\title{
MODULAR AND DECENTRALIZED PCU
}

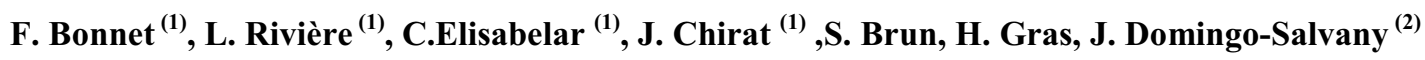 \\ (1)CNES,francois.bonnet@cnes.fr, laurent.riviere@cnes.fr,christian.elisabelar@cnes.fr \\ ${ }^{(2)}$ NEXTER Electronic,.h.gras@nexter-group.fr, j.domingo@nexter-group.fr
}

\begin{abstract}
This paper presents the studies made on the definition and design of a decentralized and modular electrical architecture of Power Conditioning Unit (PCU).

The modular PCU is able to cover a large range of mission demands by adapting the number of power modules (PM) while the electrical interfaces remain the same.

A decentralized architecture is proposed where each module is able to control the solar arrays and to manage the batteries. It appears that this kind of architecture becomes feasible thanks to digital circuits and using a communication bus [1].

Breadboards are being tested in order to validate the concept.

Reliability and robustness aspects are studied and a redundant architecture is tested.
\end{abstract}

\section{CONTEXT}

Studies made by CNES and TAS from 2008 to 2014, focused on a modular PCU based on BUCK solar array regulator with MPPT control [2]. This architecture is composed by $\mathrm{N}+1$ power modules which control the solar array, manage the battery and use a reliable digital bus for communication. This solution is compatible with unregulated or regulated busses, and the MPPT can address very large needs in spacecraft power supplies.

CNES decided to go on a second way by considering DET (Direct Energy Transfer) topologies for solar array regulator, unregulated bus and the possibility to implement remote power modules. For these reasons, this architecture is called Ultra Modular PCU. The trade-offs made for the architecture and the electronic design are more adapted for low cost applications or micro-satellites.

\section{ARCHITECTURE}

\subsection{Architecture general considerations}

The architecture is based on remote power modules connected on the same primary Unregulated Power Bus (UPB). Each module controls one or several Solar Array sections and is connected to one or several battery modules (Figure 1 and 2). A digital bus interconnects all the modules via control circuits, and is directly connected to the On-board Computer (OBC).

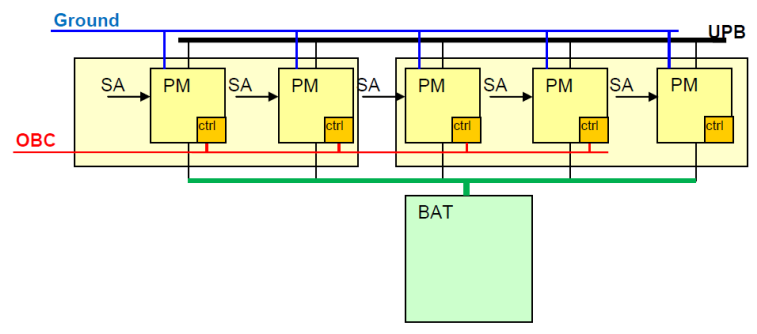

Figure 1. PCU Architecture with a single battery.

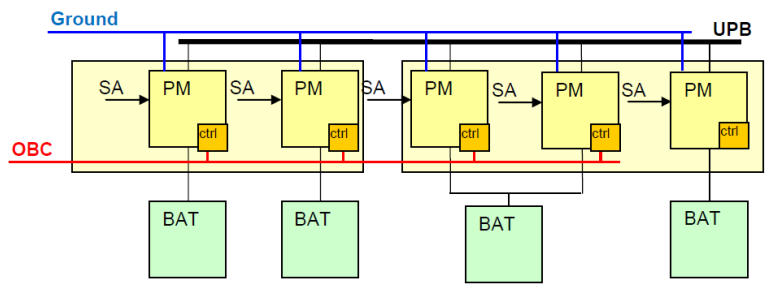

Figure 2. PCU Architecture with battery modules.

Lots of possibilities exist for the PM topology. In our objective to build a modular PCU, the elementary PM is sized for $8 \mathrm{~A}$ to $10 \mathrm{~A}$ which allows supplying a microsatellite and with $10 \mathrm{PM}$ in parallel a big LEO spacecraft can be supplied.

For example the figure 3 shows different topologies studied.

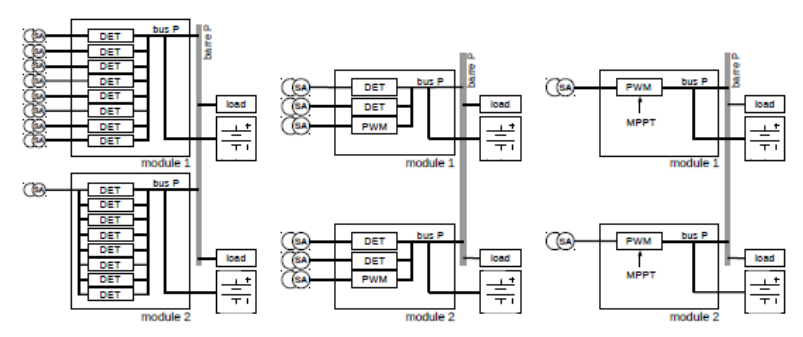

Figure 3. Some DET topologies assessed.

The first one is composed of 8 SA sections with the possibilities to connect in parallel some or all of them for modularity and adaptability aspects. Each section is sized for the total PM current divided by 8 .

Second topology uses 3 SA sections, 2 DETs and one PWM.

The third one is a DCDC converter which can operate at MPPT.

We want to keep the possibility to have remote PMs and then solutions with PWM DCDC converters (second and third examples) were not chosen because of EMC 
perturbations induced associated with the difficulty of multiple groundings of PMs.

The choice of DET with low frequency control is preferred and much more compatible with PM implemented on different places in the spacecraft.

DET topologies suppose that the SA is decomposed in several sections. This assumption is well verified because the electrical network of a SA is always made of strings with a maximum current of $2.5 \mathrm{~A}$ (with big size solar cells) and the elementary PM that we want to size is with a minimum of $10 \mathrm{~A}$ and then $4 \mathrm{SA}$ sections are needed.

The option with 8 DET is complex because the accommodation of the SA sections (parallelization) needs to configure connectors. This solution gives some constraints for the synchronization of the DET commands.

\subsection{SA regulator topologies}

The main requirements of the PM are:

- Control the operating point of the SA,

- Insure the power transfer to the battery and the spacecraft,

- Battery current and voltage regulation,

- Electrical passivation at the end of service by disconnecting the power source,

- Connect the battery to the power bus,

- Insure the full performance operation after one single failure,

- Acquisition of voltages and currents of the PM,

- Digital communication with the On Board Computer,

- ON or OFF launch.

The power of each module is also considered. The ratios of the different average powers from SA, Battery and output delivered power are summarized on table 1 .

\begin{tabular}{|c|c|}
\hline$P_{G S} / P_{s l}$ & 2.5 à 3 \\
\hline$E_{\text {batt }} / P_{s l}|\mathrm{~s}|$ & 4 à 5 \\
\hline$E_{\text {batt }} / P_{G S}|\mathrm{~s}|$ & 2 \\
\hline$E_{\text {batt }}|\mathrm{Wh}|$ & $4 \cdot V_{\text {batt }} \cdot I_{s l}$ \\
\hline
\end{tabular}

Table 1. Power ratios

An average output delivered power (Psl) of $100 \mathrm{~W}$ is chosen, which give a SA power (Pgs) from $250 \mathrm{~W}$ to $300 \mathrm{~W}$. The global SA current is then from $7 \mathrm{~A}$ to $13 \mathrm{~A}$ on a Non-Regulated Bus standard of $(24 \mathrm{~V}-38 \mathrm{~V})$.

Several topologies were assessed and finally 3 of them are short-listed for the trade-off:

- 6 configurable SA sections (Figure 5),

- 6 configurable SA sections with an additional one for redundancy (Figure 6),

- 3 reliable SA sections (Figure 7).

Shunt DET is preferred instead of serial DET (figure 4), considering transistor command (floating on serial DET) and failure cases are more advantageous for shunt DET.
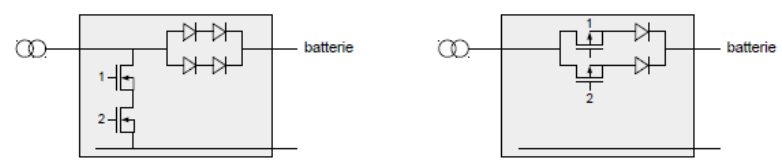

Figure 4 : Shunt DET and Serial DET

The current sharing in case of a configurable DET presents some drawbacks on the MOSFETs sizing and on their commands.

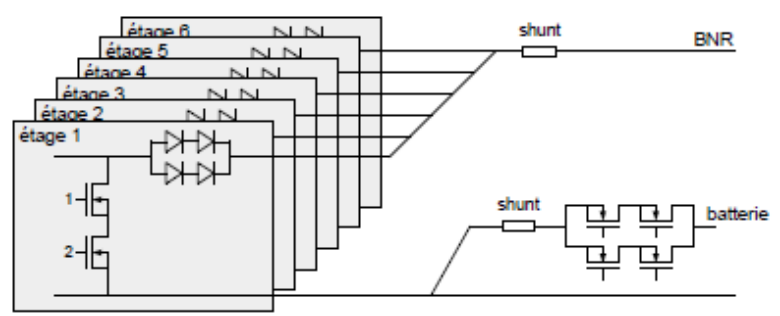

Figure 5 : 6 configurable SA sections

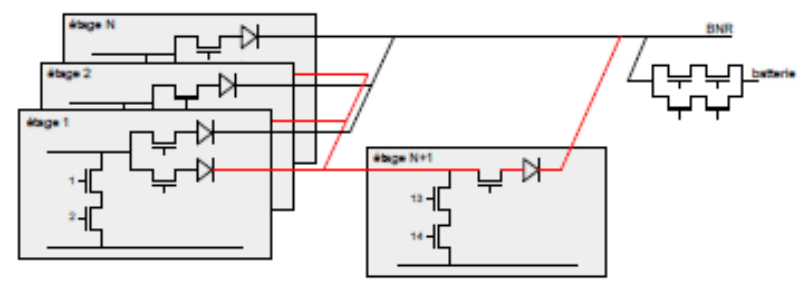

Figure 6:6+1 configurable SA sections

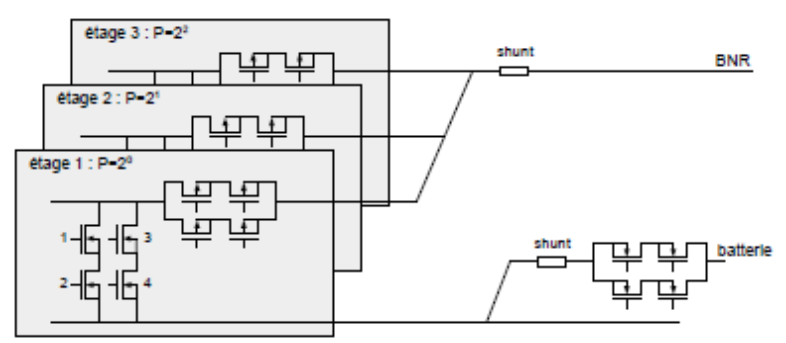

Figure 7 : 3 Reliable SA sections

On the topologies of figures 5 and 6 each SA section is sized by the current of the section, while in topology of figure 7, each SA section is sized for the global SA current delivered to the PM. 6 elementary DETs are compared with 3 oversized DETs.

\subsection{Control}

The key circuit for the control of the PM is a microcontroller. We take the option where the microcontroller is not fully rad hard and has some sensibilities regarding radiation environment. It is the 
case of some components which were evaluated at CNES and remain tolerant for cumulative dose but have some internal parts of the circuit sensitive to SEE. In order to cope with this situation and thanks to our experience on microcontrollers used in flight the following precautions are implemented:

- Corruption of the memory: the firmware rewrites sequentially the program memory, the program file is written in an EEPROM not sensitive, the microcontroller restarts in case of external watchdog triggering.

- Latch-up protection circuit is added with current protection and restart of the microcontroller.

- For the cumulated radiation dose, the components shall be rad tolerant.

If a single event effect occurs, even if the design can recover the microcontroller, the operation is interrupted during a few milliseconds. The global command of the PMs switches shall not be disturbed during these recoveries and then specific circuit shall maintain the correct statuses of the power parts.

Reliability and safety shall be also taken into account. To cope with these considerations some hardware topologies are proposed:

- 3 voted microcontrollers (Figure 8),

- 5 voted microcontrollers,

- 2 microcontrollers with reliable multiplexed DET commands with or without memorization (Figure 9),

- One microcontroller per DET switch,

- Full rad hard microcontroller.

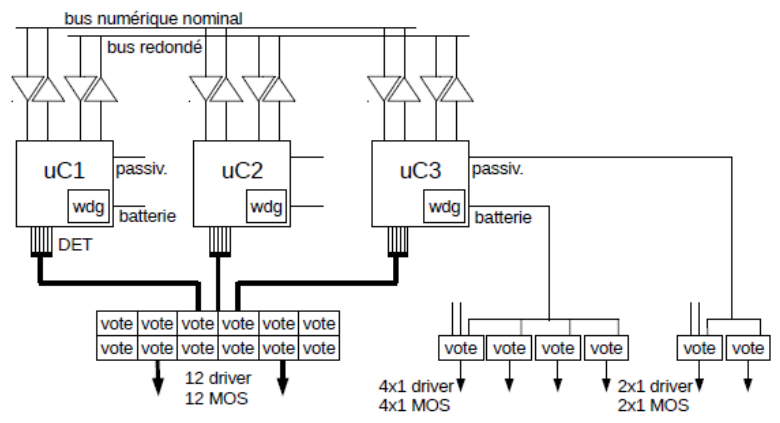

Figure $8: 3$ voted microcontrollers

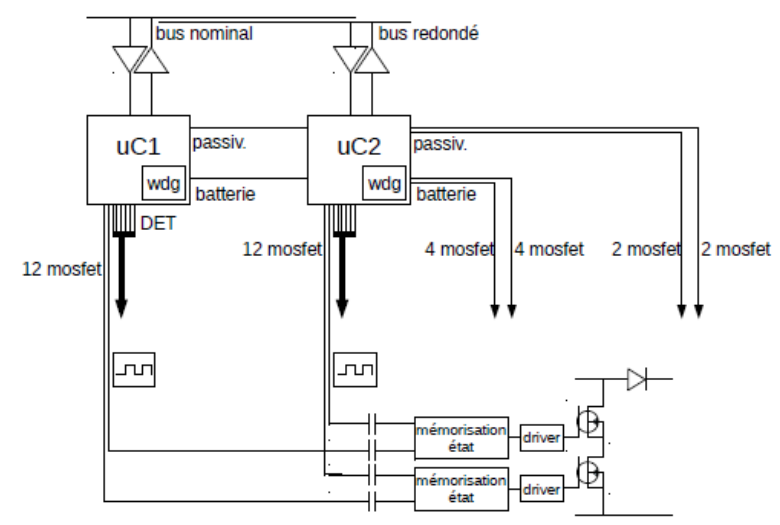

Figure 9 : 2 microcontrollers with DET commands memorization

Erroneous command (bit-flip) is also considered. This case is particularly a problem when several SA sections are connected together in parallel (case of configurable SA sections), the full SA current of the PM can run through one section. This failure case induces to oversize each SA section.

\subsection{PM topologies trade-off}

The following criteria are used for the trade-off:

- Volume,

- Number of connectors,

- Maximum delivered output power of the module,

- Efficiency (or dissipation),

- Number of power components mounted on module structure,

- Cost,

- Management of bit-flip, latch-up, CC drainsource of the MOSFETs, CC drain-gate of the MOSFETs, CC gate-source of the MOSFETs.

For the sizing aspects, it is considered that the maximum dissipated power per power component (MOSFET, diode) mounted on PCB is $500 \mathrm{~mW}$ and when mounted on module structure $10 \mathrm{~W}$.

The PM topology selected is the 3 reliable SA sections in shunt configuration, with 2 redundant microcontrollers and command memorization.

\section{BREADBOARD DESCRIPTION}

\subsection{Global breadboard description}

The breadboard is composed by 3 PM modules connected together with 9 SA sections and one battery as shown on figure 10 . 


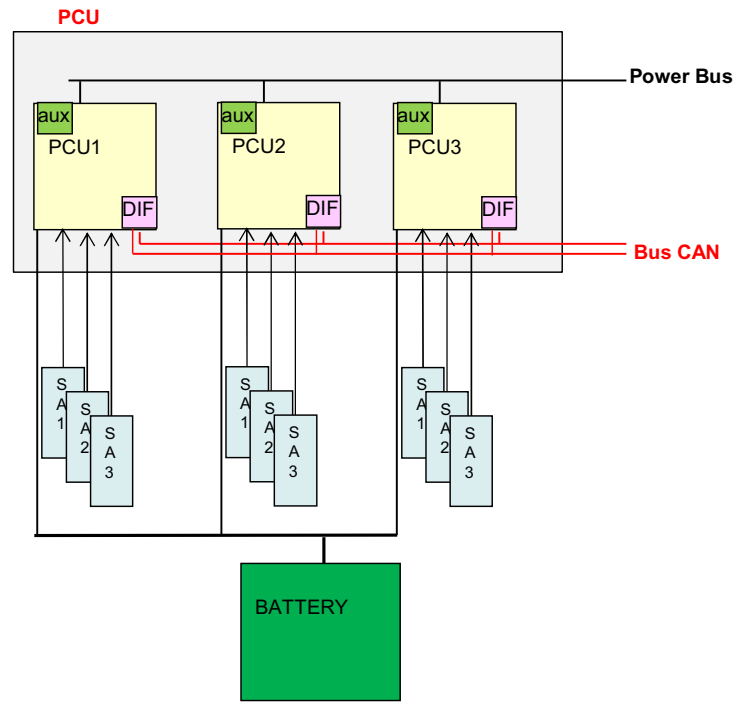

Figure $10:$ Breadboard architecture

\subsection{PM hardware}

The synoptic of the PM breadboard is given in figure 11 and compliant with the topology selected at the tradeoff.

Each shunt switch is composed by a quad of MOSFET where each branch is controlled by the same microcontroller (branch A (B) controlled by microcontroller A (B).

On the breadboard the serial switch is composed of a quad of diodes. Other solutions using MOSFETs are studied and could be preferably implemented but not for this breadboard. The main objective here remains to test functional aspects instead of efficiency performances on real power condition.

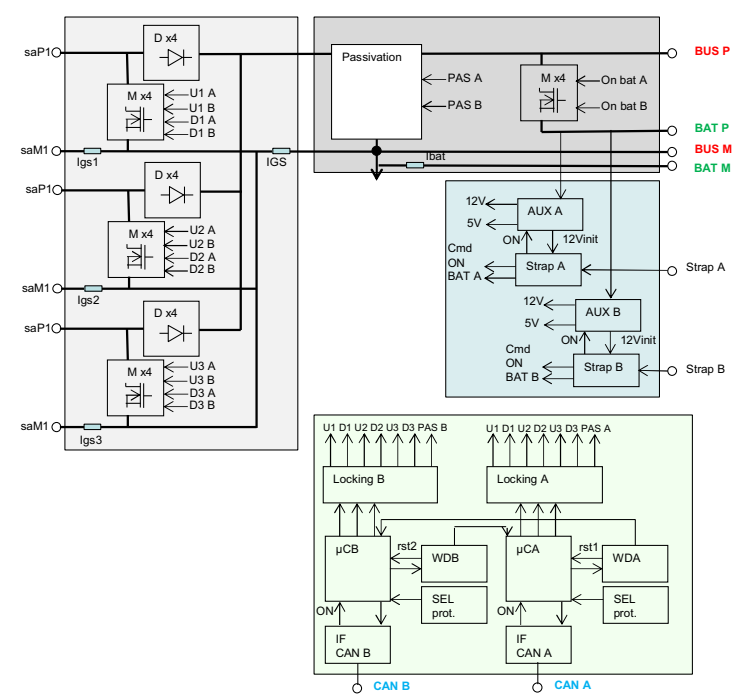

Figure 11 : PM breadboard hardware synoptic

The connection with the battery or battery module is performed by a quad of MOSFETs.
A specific passivation circuit allows disconnecting the SA regulator from the other parts of the PM.

Redundant auxiliary supplies give the secondary voltages to supply all the module circuits.

A strap detection circuit maintains each auxiliary supply OFF during launch (in launch OFF configuration) and at the same time gives polarisations to shunt all the DETs. When the separation is detected, the auxiliary supplies start nominally, and switch ON the battery then the PCU starts.

The global control of the power module is done by 2 redundant digital circuits. Each one is composed of a microcontroller, a rad-hard watchdog, a latch-up protection, a rad-hard locking device and a CAN transceiver.

The breadboard is reduced power model with a bus voltage of $30 \mathrm{~V}$ and a SA current per section of $100 \mathrm{~mA}$.

\subsection{PM Software}

The system operates in hot redundancy where the two chains execute the same firmware.

Some of the parameters used by the PCU software are stored in EEPROM memory: ID of the PCU module, battery voltage setting for the regulation algorithm, maximum battery current allowed, allocation table used by the sections allocation algorithm, array with the address of the sections grouped in the allocation table.

The initialization process selects the clock frequency, the inputs / outputs, initializes the operational mode, loads the EEPROM parameters, initializes the CAN bus functions, the $\mathrm{ADC}$, the timer, the regulation algorithm, and the commands.

The PCU can be in different working modes. A working mode corresponds to a sequence of actions which are executed each $1 \mathrm{~s}$.

- Operational mode is the main mode of the PM. Acquisitions are shared between the PMs, the regulation is working and OBC commands are processed. This working mode is automatically operational after starting or after a reset.

- Passivation mode is used to receive and start arming/passivation commands. In this mode, all the SA sections are commanded shunted.

- Failure mode is used when an error occurs mainly during EEPROM parameters reading. In this mode, all the DET shunt switches are opened to let the redundant line operate. 


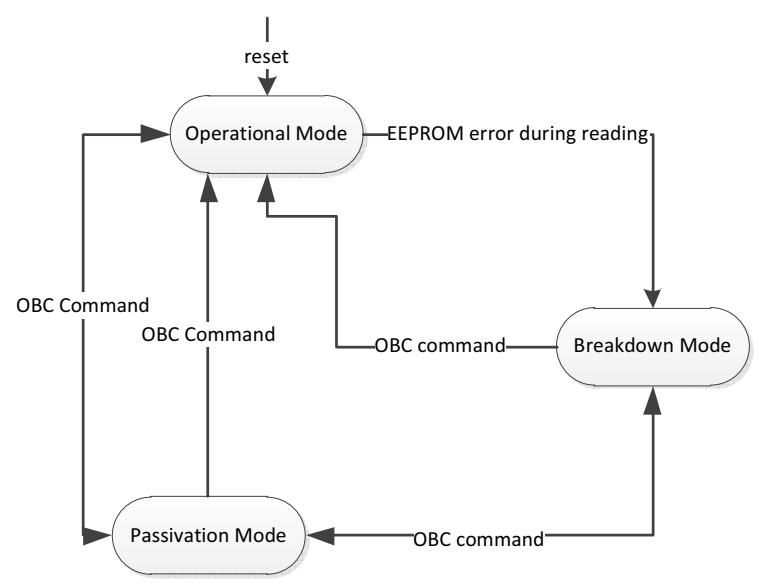

Figure 12 : Software modes

Operational mode is composed of 2 parts:

- The regulation process: acquisitions are done and shared between all the PMs. The regulation algorithm is executed and the sections are closed or opened using the allocation table. Service messages are sent (PM statuses, sections currents and voltage values).

- The commands process: Commands from the OBC are monitored and processed.

Failure mode is composed of 2 parts:

- The acquisition process: acquisitions are done and shared between all the PMs. All DET shunts are opened to let the redundant line performing its actions. Then, service messages are sent.

- The commands process: Commands from the OBC are monitored and processed.

Each PM executes its sequence of actions every 1s. This period of $1 \mathrm{~s}$ is defined by a timer which is used by the $\mathrm{PM}$ as a counter. The timer is based on the internal oscillator of the microcontroller which works at a frequency of $8 \mathrm{MHz}$. Because the internal oscillators of the PMs are not perfectly equal, a way to synchronize the PCU is needed. A specific algorithm combined with the CAN bus protocol is implemented to insure the synchronization of all the PMs.

The regulation algorithm computes the current setting to be provided by the solar generators. It also implements the allocation algorithm in charge of determining the set of SA sections to be activated to reach the current setting computed by the regulation algorithm.

The regulation algorithm is composed of 2 loops:

- The internal regulation loop is in charge of the regulation of the battery current delivered by the all PMs. This regulation is done by an integral corrector. Anti-windup scheme is used to prevent integration wind-up: limitation of the error during eclipse period for example.

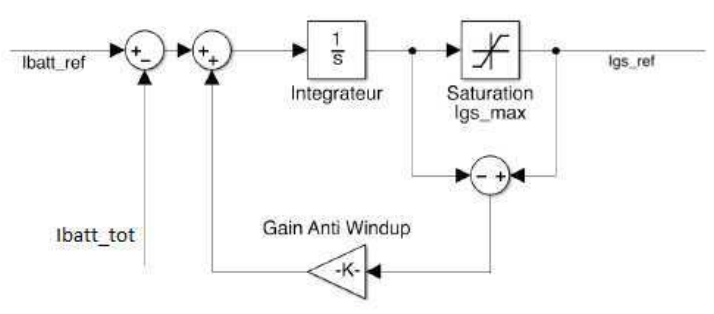

Figure 13 : Internal regulation loop

- The external regulation loop is in charge of the regulation of the battery voltage. This regulation is done by a proportional-integral corrector. The following figure presents the regulation used by each PM:

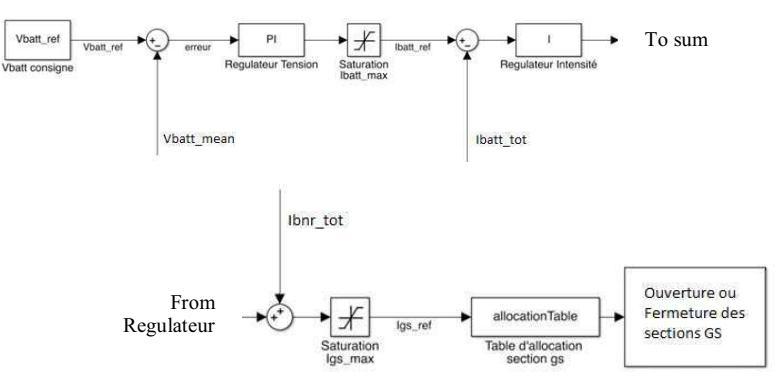

Figure 14 : External regulation loop

The average bus voltage measured by all the PMs is computed before regulation loop and for the currents it is the sum of output currents and the sum of battery currents which are processed.

The allocation table is used to choose which combinations of SA sections have to be opened or closed to reach the current setting computed by the regulation algorithm.

The table groups the short-circuit current of all SA sections of the system.

\begin{tabular}{|c|c|c|c|c|}
\hline allocationTable[0] & 150 & $\rightarrow$ & sectionIDvector[0] & PCU3 Section2 \\
\hline allocationTable[1] & 150 & $\rightarrow$ & sectionIDvector [1] & PCU3 Section 3 \\
\hline allocationTable[2] & 75 & $\rightarrow$ & sectionIDvector[2] & PCU1 Section 3 \\
\hline allocationTable[3] & 75 & $\rightarrow$ & sectionIDvector[3] & PCU3 Sectionl \\
\hline allocationTable[4] & 50 & $\rightarrow$ & sectionIDvector [4] & PCU2 Sectionl \\
\hline allocationTable[5] & 50 & $\rightarrow$ & sectionIDvector[5] & PCU2 Section2 \\
\hline allocationTable[6] & 50 & $\rightarrow$ & sectionIDvector[6] & PCU2 Section3 \\
\hline allocationTable[7] & 37.5 & $\rightarrow$ & sectionIDvector[7] & PCU1 Sectionl \\
\hline allocationTable[8] & 37.5 & $\rightarrow$ & sectionIDvector [8] & PCU1 Section2 \\
\hline
\end{tabular}

In order to reach the current setting, the short-circuit currents are added successively starting with the highest 
current (beginning of the allocation table). A section is activated if it permits to get closer to the setting.

The allocation table is very important for modularity or mission adaptation. It is in the table that it is configured the total number of SA sections with their current capabilities.

Others allocation table can be programmed in order to optimize some particular performance of the PCU. For example priority could be given for selected SA sections to switch at working frequency while others sections are only commanded from time to time.

The OBC interface provides a way to monitor acquisitions values: SA section currents, battery voltages and currents, power bus voltages, switches statuses and active working mode of each PM.

Once the acquisitions have been shared between all the PMs, each PM computes the following values in order to execute the same regulation algorithm:

- mean value of the bus voltage acquisitions,

- sum of the battery current acquisitions,

- sum of the SA sections current acquisitions,

- computed output current.

The OBC can send commands to start Arming/Passivation process (only in Passivation working mode), modify the working mode of the PM, modify regulation parameters and command SA section measurements.

\subsection{Robustness considerations}

The reliability and robustness of the system are studied. First of all power circuits (SA sections, battery interface), digital control circuits, and communication bus were redounded. With a focus on low cost the use of a COTS microcontroller can be decided, then protection circuits against SEL are implemented. Logic circuits insensitive to radiation allow monitoring of the microcontrollers, recovery and voting the SA sections orders. The two CAN buses work in hot redundancies.

Because of the topology where each branch of the shunt is controlled by one command way, the only case which can cause the loss of the power bus generation is where the 2 MOSFETs of the same branch are commanded ON permanently. This situation can occur in the event of a failure on voltage or current measurement that increases the value too high and then the regulation forces the shunting. This situation can be detected by the firmware by implementing a specific monitoring algorithm.

In the end, the $\mathrm{OBC}$ is in charge of monitoring the PCU thanks to the telemetries and can detect any failures or drifts which could occur and then is able to reconfigure the PMs.
The PCU can operate only with one way (A or B), but in this failure case some short interruptions no longer than 1s can occur if one microcontroller is restarted. This situation is not a problem because the nonregulated bus concept can insure a permanent supply of the spacecraft, and also because the commands of the DETs are locked at the previous states during recovery.

\section{EXPERIMENTAL RESULTS}

The figure 15 shows the general experimental setup. The $3 \mathrm{PM}$ are stacked together and connected to $9 \mathrm{SA}$ simulators and one Li-Ion battery.

The breadboard is a reduced power model with a capability of $200 \mathrm{~mA}$ per SA section. A SA simulator composed of 24 sections of $100 \mathrm{~mA}$ has been developed for these activities. The battery is composed by one string of 8 Li-Ion cells. The power bus is loaded by a resistance and an active load.

The digital CAN busses are connected to a PC simulating the $\mathrm{OBC}$ and allowing the display on an MI of all the date exchanges on the 2 redundant CAN buses.

The system operate at $1 \mathrm{~Hz}$ : all the $\mathrm{SA}$ sections status are refreshed each second.

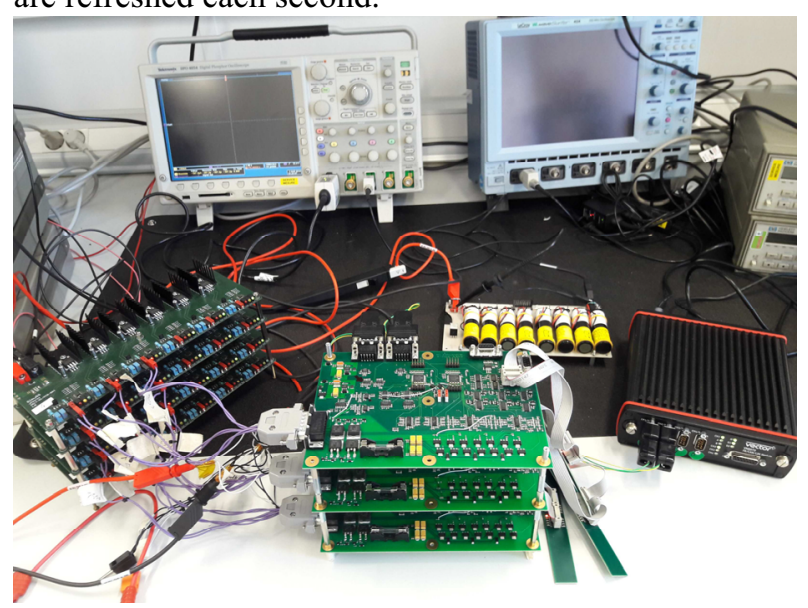

Figure 15. The 3 PMs during test.

Figure 16 shows the recharge of the battery, at the beginning the battery current is limited and then when the bus voltage reaches the limitation reference at $30.4 \mathrm{~V}$, the current in the battery decrease. The ripple on the voltage is directly due to the DC impedance of the battery multiplied by the current switching steps. 


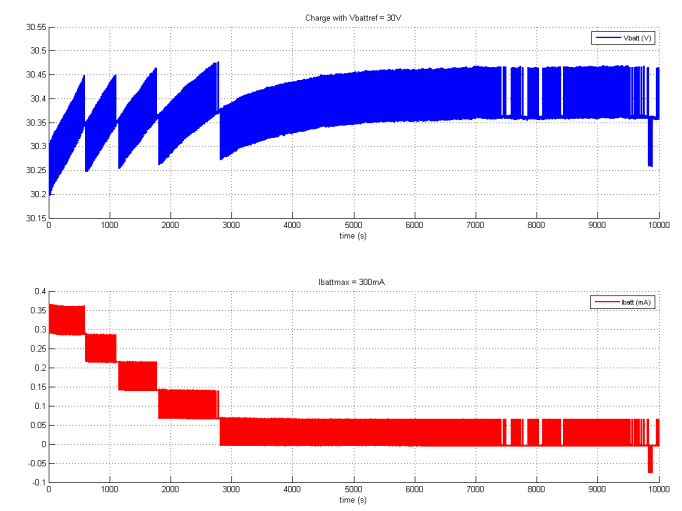

Figure 16. Experiment results, bus voltage (top) and battery current (down).

\section{CONCLUSION AND FUTURE WORKS}

The architecture of a decentralized and modular PCU gives performances as expected. Thanks to the digital control, lots of possibilities are offered to control the SA regulation or the battery management, and the capability to customize the PCU operation. Architecture and circuits design implementation are compliant with reliability and robustness needs and the system will be single failure free.

Future works will focus on achieving a real power model including all the redundancies and a spatial design compatible. Moreover, control laws improvements such as FDIR, robustness improvements, are still in progress.

The hardware architecture will remain decentralized, and the software architecture too. But with the same hardware, centralized software architecture is feasible where all the commands and control are made by the On Board Computer. In this case, each PM firmware keeps only the execution of the OBC commands, the TM acquisitions and the dialog with the OBC. It is very similar to PROTEUS or PLEÏADES PCU, but here there is modularity and the algorithms developed have lots of possibilities: adaptation with the number of PMs, allocation table for better regulation performances.

\section{REFERENCES}

1. C.Elisabelar, L. Lebaratoux : "Decentralized and Modular electrical architecture" ESPC2014.

2. M.Fossion, V. Lempereur, S. Fredon, C. Elisabelar, J.M. Cresp : «A modular and digitally controlled power conditioning unit” ESPC 2011. 